\title{
THE CYCLIC JACOBI METHOD FOR COMPUTING THE PRINCIPAL VALUES OF A COMPLEX MATRIX $\left({ }^{(}\right)$
}

BY

\author{
G. E. FORSYTHE AND P. HENRICI
}

\section{INTRODUCTION AND SUMMARY}

1.1. Jacobi's method for computing the eigenvalues of a real symmetric matrix. Let $A=\left(a_{p q}\right)$ be a real symmetric matrix of order $n$, and let $\lambda_{1}, \lambda_{2}, \cdots, \lambda_{n}$ be its eigenvalues. It is well known that if $U$ is an orthogonal matrix such that

$$
\Lambda=U A U^{T}
$$

is diagonal ( $T$ denotes the transpose), then the main diagonal of $\Lambda$ is made up of the numbers $\lambda_{i}$ in some order. If it is desired to compute the $\lambda_{i}$ numerically, this result is of no immediate use, since for $n>2$ there exists no manageable expression for the general orthogonal matrix of order $n$. However, Jacobi [6] suggested the computation of the set of $\lambda_{i}$ as the limiting set of diagonal elements of a sequence of matrices which are generated from $A$ recursively by plane rotations.

For $k=0,1,2, \cdots$, let $\phi=\phi_{k}$ be a real angle and $(i, j)=\left(i_{k}, j_{k}\right)$, a pair of integers such that $1 \leqq i_{k}<j_{k} \leqq n$. The matrix $U_{k}=\left(u_{p q}\right)$, where

$$
\begin{aligned}
u_{p p} & =1 & & \\
u_{i i} & =\cos \phi, & u_{i j} & =\sin \phi, \\
u_{j i} & =-\sin \phi, & u_{j j} & =\cos \phi, \\
& & \text { all other } u_{p q} & =0,
\end{aligned}
$$$$
(p \neq i, j)
$$

is clearly orthogonal. We define a sequence of matrices $A_{k}=\left(a_{p q}^{(k)}\right)$ by

$$
A_{0}=A, \quad A_{k+1}=U_{k} A_{k} U_{k}^{T} \quad(k=0,1,2, \cdots) .
$$

The eigenvalues of $A_{k}$ are the same as those of $A$. Hence, if

$$
\lim _{k \rightarrow \infty} A_{k}=\Lambda
$$

exists and is diagonal, or if there exist permutation matrices $P_{k}$ such that

Presented to the Society, December 27, 1956 under the title Proof of convergence of the cyclic Jacobi iterative process for computing eigenvalues of a real symmetric matrix; received by the editors March 3, 1957 and, in revised form, June 8, 1958.

(1) This work was sponsored by the Office of Naval Research, U. S. Navy, and by the Office of Ordnance Research, U. S. Army. Reproduction in whole or in part is permitted for any purpose of the United States Government. 


$$
\lim _{k \rightarrow \infty} P_{k} A_{k} P_{k}^{-1}=\Lambda
$$

exists and is diagonal, then $\Lambda$ has on its diagonal the eigenvalues of $A$. Jacobi essentially showed that (4) holds if the matrices $U_{k}$ are selected as follows: Choose

$$
\left(i_{k}, j_{k}\right) \text { such that }\left|a_{i j}^{(k)}\right|=\max _{p \neq q}\left|a_{p q}^{(k)}\right|
$$

and

$$
\phi_{k} \text { such that } a_{i j}^{(k+1)}=0 .
$$

He also showed that (II) can always be realized and gave an estimate for the rate of convergence of the method. In [3] Goldstine, Murray, and von Neumann gave a description of Jacobi's method and studied the effect of roundoff errors.

1.2. Extensions of Jacobi's method. In this paper we extend and generalize Jacobi's method in various directions. We shall first describe the results which we obtain in each of these directions, and then in $\$ 1.3$ synthesize them in the form of four theorems. While one of these extensions is in common use on automatic digital computers, ours is apparently the first proof of its convergence.

The cyclic Jacobi method. Jacobi's method in its original form requires at each step the scanning of $n(n-1) / 2$ numbers for one of maximum modulus. For large matrices this is a relatively slow process, especially for automatic digital computers. It is more convenient to select the pairs $(i, j)$ in some cyclic order. We here consider two cyclic orders: (i) cyclic by rows, indicated by the scheme

$\left(i_{k+1}, j_{k+1}\right)= \begin{cases}\left(i_{k}, j_{k}+1\right), & \text { if } i_{k}<n-1, j_{k}<n, \\ \left(i_{k}+1, i_{k}+2\right), & \text { if } i_{k}<n-1, j_{k}=n, \\ (1,2), & \text { if } i_{k}=n-1, j_{k}=n ;\end{cases}$

and (ii) cyclic by columns, as follows:

$$
\begin{aligned}
\left(i_{0}, j_{0}\right) & =(1,2), \\
\left(i_{k+1}, j_{k+1}\right) & = \begin{cases}\left(i_{k}+1, j_{k}\right), & \text { if } i_{k}<j_{k}-1, j_{k} \leqq n, \\
\left(1, j_{k}+1\right), & \text { if } i_{k}=j_{k}-1, j_{k}<n, \\
(1,2), & \text { if } i_{k}=n-1, j_{k}=n .\end{cases}
\end{aligned}
$$

A modified Jacobi method, here called the cyclic Jacobi method, consisting of selecting $\left(i_{k}, j_{k}\right)$ according to one of the rules (III) and $\phi_{k}$ according to (II), is used on electronic computers (see Gregory [5]), apparently with 
satisfactory results. Using both analysis and machine experiments, Pope and Tompkins [12] have studied the convergence of certain variations of the Jacobi method, including one in which the rotations are not performed unless $\left|\phi_{k}\right|$ exceeds a prescribed threshold value. Givens [2] has used the rotations (2) in a different way to bring $A$ in one cycle to an orthogonally congruent triple diagonal form, and has discussed the round-off error in detail. An attempt to prove the convergence of $A_{k}$ to $\Lambda$ and the analogous result for principal values stated below, based on heuristical statistical arguments, has been published by Kogbetliantz [7].

We shall exhibit examples which show that under either rule (III) convergence in the sense of (4) cannot be guaranteed if $\phi_{k}$ is subjected solely to the condition (II). On the other hand it will be shown that convergence in the stronger sense of (3) does take place if the $\phi_{k}$ are subjected to the further restriction that

$$
\left.\phi_{k} \in J \quad \text { (for all } k=0,1,2, \cdots\right),
$$

where $J$ is some closed interval independent of $k$ and interior to the open interval $(-\pi / 2, \pi / 2)$. It will also be shown that the conditions (II) and (IV) can always be realized simultaneously.

Eigenvalues of hermitian matrices. If $A$ is a hermitian matrix, and if $U$ is a unitary matrix such that $\Lambda=U A U^{*}$ is diagonal (* denotes the complex conjugate of the transpose), then as before the main diagonal of $\Lambda$ consists of the eigenvalues of $A$ in some order. It is therefore natural to ask whether either the original or the cyclic Jacobi method can be extended to hermitian matrices by replacing the submatrices

$$
\left(\begin{array}{rr}
\cos \phi_{k} & \sin \phi_{k} \\
-\sin \phi_{k} & \cos \phi_{k}
\end{array}\right)
$$

of the matrices $U_{k}$ defined in $\S 1.1$ by suitably chosen $2 \times 2$ unitary matrices. This has already been done formally by Kogbetliantz [7]. Greenstadt [4] and Lotkin [9] have proposed other methods for the computation of the eigenvalues of nonsymmetric matrices which use $2 \times 2$ unitary transformations. All these authors chose their unitary matrices to be of some special type.

In contrast to this, we shall not subject the unitary matrices involved to any condition not imposed by the problem itself. It is easy to see that any $2 \times 2$ unitary matrix can be represented in the form

$$
\left(\begin{array}{rr}
e^{i \alpha} \cos \phi & e^{i \beta} \sin \phi \\
-e^{i \gamma} \sin \phi & e^{i \delta} \cos \phi
\end{array}\right)
$$

where $\alpha, \beta, \gamma, \delta, \phi$ are real numbers with

$$
\alpha-\beta-\gamma+\delta \equiv 0(\bmod 2 \pi) \text {. }
$$


(Causey [1] has given an equivalent representation of $2 \times 2$ unitary matrices.) Consequently we shall consider unitary matrices $U_{k}=\left(u_{p q}\right)$ which are of the form $\left({ }^{2}\right)$

$$
\begin{array}{rlrl}
u_{p p} & =1 \\
u_{i i} & =e^{i \alpha} \cos \phi, & & \\
u_{j i} & =-e^{i \gamma} \sin \phi, \quad e^{i \beta} \sin \phi, \\
u_{j j} & =e^{i \delta} \cos \phi, \\
& \text { all other } u_{p q} & =0,
\end{array}
$$

where $\alpha, \beta, \gamma, \delta, \phi$ are real numbers satisfying (5) and depending on $k$.

We shall show for Jacobi's rule (I) and for either of the cyclic orders (III) that the sequence of matrices

$$
A_{0}=A, \quad A_{k+1}=U_{k} A_{k} U_{k}^{*} \quad(k=0,1,2, \cdots)
$$

converges to a diagonal matrix for all choices of matrices $U_{k}$ of the form (6) satisfying (II) and (IV) with respect to $\phi$.

It will be shown that matrices $U_{k}$ with these properties always exist.

Moreover, for Jacobi's rule (I) - but not for a cyclic order (III) - we shall show that there exists a sequence of permutation matrices $P_{k}$ such that $P_{k} A_{k} P_{k}^{-1}$ converges to a diagonal matrix for all choices of matrices $U_{k}$ of the form (6) satisfying (II) with respect to $\phi$, and not necessarily satisfying (IV).

Principal values of arbitrary matrices. In spite of certain attempts $[4 ; 9 ; 1]$, there does not seem to be an obvious way of modifying Jacobi's method to yield the eigenvalues of a nonhermitian matrix. As Kogbetliantz [7] states, however, it is easy to extend Jacobi's method formally to yield the principal values of an arbitrary complex matrix $A$. By the principal values of $A$ we mean the positive square roots of the eigenvalues of $A A^{*}$. (These numbers are occasionally called the singular values of $A$.) The extension is based on the following result (see [10, Corollary 41.6]): If $U$ and $V$ are two unitary matrices such that $\Pi=U A V$ is diagonal, then, since $\Pi \Pi^{*}=U A A^{*} U^{*}$, the absolute values of the diagonal elements of $\Pi$ are the principal values of $A$.

For $k=0,1,2, \cdots$ let $U_{k}$ be a matrix of the form (6), and let $V_{k}=\left(v_{p q}\right)$ be defined similarly by

$$
\begin{array}{rlrl}
v_{p p} & =1 \\
v_{i i} & =e^{i \xi} \cos \psi, & v_{i j} & =e^{i \eta} \sin \psi, \\
v_{j i} & =-e^{i \zeta} \sin \psi, \quad v_{j j} & =e^{i \omega} \cos \psi, \\
\text { all other } v_{p q} & =0 ;
\end{array}
$$$$
(p \neq i, j),
$$

here $\xi, \eta, \zeta, \omega, \psi$ are real numbers depending on $k$, the first four of which satisfy the condition

(2) Here and below $i$ as a superscript to $e$ denotes $(-1)^{1 / 2}$, while in other contexts $i$ is a row or column index. 


$$
\xi-\eta-\zeta+\omega \equiv 0(\bmod 2 \pi) \text {. }
$$

We now consider the sequence of matrices $A_{k}=\left(a_{p q}^{(k)}\right)$, where

$$
A_{0}=A, \quad A_{k+1}=U_{k} A_{k} V_{k} \quad(k=0,1,2, \cdots) .
$$

Let $\left|A_{k}\right|=\left(\left|a_{i j}^{(k)}\right|\right)$ denote the matrix of non-negative elements $\left|a_{i j}^{(k)}\right|$, and not the determinant of $A_{k}$.

It will be shown that the sequence $\left\{\left|A_{k}\right|\right\}$ converges to a diagonal matrix whose diagonal elements are the principal values of $A$, if one of the following two procedures is adopted:

(i) Select $\left(i_{k}, j_{k}\right)$ such that

$$
\left|a_{i j}^{(k)}\right|^{2}+\left|a_{j i}^{(k)}\right|^{2}=\max _{p \neq q}\left\{\left|a_{p q}^{(k)}\right|^{2}+\left|a_{q p}^{(k)}\right|^{2}\right\}
$$

and the remaining parameters of $U_{k}$ and $V_{k}$ such that

$$
a_{i j}^{(k+1)}=a_{j i}^{(k+1)}=0
$$

and such that $\phi_{k}$ and $\psi_{k}$ satisfy the conditions

$$
\phi_{k} \in J, \quad \psi_{k} \in J
$$$$
\text { (all } k=0,1,2, \cdots) \text { ), }
$$

where $J$ is some closed interval independent of $k$ and interior to the open interval $(-\pi / 2, \pi / 2)$.

(ii) Select $\left(i_{k}, j_{k}\right)$ according to either $\left(\mathrm{III}_{r}\right)$ or $\left(\mathrm{III}_{c}\right)$, and the remaining parameters such that $\left(\mathrm{II}^{\prime}\right)$ and $\left(\mathrm{IV}^{\prime}\right)$ hold.

It will moreover be proved that there exists a sequence of permutation matrices $P_{k}$ such that $P_{k}\left|A_{k}\right| P_{k}^{-1}$ converges to a diagonal matrix whose diagonal elements are the principal values of $A$, provided that $\left(i_{k}, j_{k}\right)$ is selected according to $\left(\mathrm{I}^{\prime}\right)$, and provided that the remaining parameters of $U_{k}$ and $V_{k}$ are selected to satisfy $\left(\mathrm{II}^{\prime}\right)$.

It will be demonstrated that the conditions given under (i) can always be realized. The conditions $\left(\mathrm{II}^{\prime}\right)$ and $\left(\mathrm{IV}^{\prime}\right)$, on the other hand, cannot be realized simultaneously in certain cases, so that convergence of the cyclic Jacobi method for determining principal values in the above form cannot be guaranteed. This situation will be remedied by a device to be explained next.

Under-or overrotation. It will finally be shown that it is not necessary to take conditions (II) and ( $\left.\mathrm{II}^{\prime}\right)$ too literally. All statements made above remain valid if these conditions are replaced by the following weaker conditions: There exists a number $t(0 \leqq t<1)$ independent of $k$ such that for $k=0,1,2, \ldots$

$$
\left|a_{i j}^{(k+1)}\right|^{2} \leqq t\left|a_{i j}^{(k)}\right|^{2}
$$

and

$$
\left|a_{i j}^{(k+1)}\right|^{2}+\left|a_{j i}^{(k+1)}\right|^{2} \leqq t\left(\left|a_{i j}^{(k)}\right|^{2}+\left|a_{j i}^{(k)}\right|^{2}\right),
$$


respectively. The fact that the weaker conditions $(\mathrm{V})$ and $\left(\mathrm{V}^{\prime}\right)$ are sufficient for convergence is important for several reasons. First, it can be shown that (in contrast to $\left(\mathrm{II}^{\prime}\right)$ ) condition $\left(\mathrm{V}^{\prime}\right)$ can be realized simultaneously with $\left(\mathrm{IV}^{\prime}\right)$ for every $t>0$. Second, the necessary rounding off of numbers in a digital computer means that conditions (II) and $\left(\mathrm{II}^{\prime}\right)$ can never be achieved exactly, while $(\mathrm{V})$ and $\left(\mathrm{V}^{\prime}\right)$ certainly can. Third, the use of a $t>0$ corresponds to an under- or overrotation of the $\left(i_{k}, j_{k}\right)$ coordinate plane. Its use here brings out the analogy between rotation in the various Jacobi methods for computing eigenvalues or principal values and relaxation in the methods of Gauss, Seidel, and Southwell for the iterative solution of a system of linear algebraic equations. See Ostrowski [11] for a discussion of under- and overrelaxation for linear systems.

1.3. Summary of the results of this paper. In the general case we are given an arbitrary complex matrix $A$ of order $n \geqq 2$ and two sequences of unitary matrices $\left\{U_{k}\right\}$ and $\left\{V_{k}\right\}$ defined by (6) and (7). Consider the sequence of matrices $A_{k}=\left(a_{p q}^{(k)}\right)$ defined by

$$
A_{0}=A, \quad A_{k+1}=U_{k} A_{k} V_{k} \quad(k=0,1,2, \cdots) .
$$

Let $I I$ be a diagonal matrix whose diagonal elements are the principal values of $A$ in some preassigned fixed order. If $A$ is hermitian, let $\Lambda$ be the diagonal matrix whose diagonal elements are the eigenvalues of $A$ in some preassigned fixed order. We are interested in sufficient conditions under which the following proposition is true:

Proposition (N). Let $\left|A_{k}\right|=\left(\left|a_{p q}^{(k)}\right|\right)$. There exists a sequence of permutation matrices $P_{k}$ such that

$$
P_{k}\left|A_{k}\right| P_{k}^{-1} \rightarrow \mathrm{II} \quad(k \rightarrow \infty) .
$$

If $A$ is hermitian and $V_{k}=U_{k}^{*}$, there exists a sequence of permutation matrices $P_{k}$ such that

$$
P_{k} A_{k} P_{k}^{-1} \rightarrow \Lambda \quad(k \rightarrow \infty) .
$$

We are also interested in conditions for the following stronger proposition: $\left(^{3}\right)$

Proposition (P). There exists a fixed permutation matrix $P$ (not depending on $k$ ) such that

$$
P\left|A_{k}\right| P^{-1} \rightarrow \Pi \quad(k \rightarrow \infty) .
$$

If $A$ is hermitian and $V_{k}=U_{k}^{*}$, there exists a fixed permutation matrix $P$ such that

$$
P A_{k} P^{-1} \rightarrow \Lambda \quad(k \rightarrow \infty) .
$$

(3) We are indebted to the referee for pointing out the distinction between Propositions $(\mathrm{N})$ and $(\mathrm{P})$, and for showing us the example (41) of $\$ 2.4$. 
Introducing the abbreviation

$$
s_{p q}^{(k)}=\left|a_{p q}^{(k)}\right|^{2}+\left|a_{q p}^{(k)}\right|^{2},
$$

we can state our main results as follows:

Theorem 1. (JACOBI'S MEthod Generalized.) Let $t$ be independent of $k$, with $0 \leqq t<1$. Then Proposition $(\mathrm{N})$ is true if for all large $k$

$$
s_{i j}^{(k)}=\max _{p \neq q} s_{p q}^{(k)},
$$

and

$$
s_{i j}^{(k+1)} \leqq t s_{i j}^{(k)} .
$$

Theorem 2. Let $J$ be a closed interval interior to the open interval $(-\pi / 2$, $\pi / 2)$. If, in addition to the conditions of Theorem 1 ,

$$
\phi_{k} \in J, \quad \psi_{k} \in J
$$

for all large $k$, then Proposition $(\mathrm{P})$ is true.

Theorem 3. (The cyclic Jacobi method generalized.) Let $t$ and $J$ be defined as in Theorems 1 and 2 . Let the sequence of pairs $\left(i_{k}, j_{k}\right)$ be defined by one of the rules (III). Then Proposition (P) is true if $\left(\mathrm{V}^{\prime}\right)$ and $\left(\mathrm{IV}^{\prime}\right)$ hold for all large $k$. Even Proposition $(\mathrm{N})$ becomes false if $\left(\mathrm{IV}^{\prime}\right)$ is omitted from the hypothesis.

The proofs of these theorems will be given in $\$ 2$. We shall begin by proving Theorem 1 and a weaker form of Theorem 3, which is obtained from Theorem 3 by replacing $(\mathrm{P})$ by $(\mathrm{N})$. Theorem 2 and the full statement of Theorem 3 are then a consequence of the following Theorem 4 , in which no reference is made to any particular ordering of the pairs $\left(i_{k}, j_{k}\right)$.

THEOREM 4. Any choice of the matrices $U_{k}$ and $V_{k}$ (or, for hermitian A, of the $U_{k}$ ) which implies the truth of Proposition (N) and satisfies (IV') (or, in the case of hermitian A, (IV)), also implies the truth of Proposition (P). Proposition $(\mathrm{P})$ is not necessarily true if $\left(\mathrm{IV}^{\prime}\right)$ is not satisfied.

In $\$ 3$, we shall derive explicit formulas for the parameters involved in $U_{k}$ and $V_{k}$. They will enable us to discuss how the hypotheses on $U_{k}$ and $V_{k}$ can be realized in the various cases mentioned in $\$ 1.2$.

\section{Proof of the theorems of $\$ 1$}

2.1. Preliminary lemmas. Following Jacobi, we take as a measure of the closeness of the matrix $\left|A_{k}\right|$ (or $A_{k}$ ) to a diagonal matrix the non-negative quantity 


$$
S^{(k)}=\sum_{p \neq q}\left|a_{p q}^{(k)}\right|^{2}
$$

The relation

$$
\lim _{k \rightarrow \infty} S^{(k)}=0
$$

is evidently necessary for Proposition (N). The plan of our proofs is as follows: We show in $\$ 2.1$ that the conditions of Theorem 1 imply (11). In $\$ 2.2$ we prove that the conditions of Theorem 3 imply (11). We then proceed to show in $\$ 2.3$ that (11) is in fact equivalent to Proposition $(\mathrm{N})$, and this proves Theorem 1 . Theorem 4 is proved in $\$ 2.4$, whence follow Theorems 2 and 3 . In $\$ 2.5$ we show that $\left(I V^{\prime}\right)$ is necessary as well as sufficient for the validity of Theorem 3 .

In the following discussion we shall frequently write

$$
a_{p q}^{(k)}=a_{p q}, \quad a_{p q}^{(k+1)}=a_{p q}^{\prime} .
$$

A similar notation may be used for other quantities which depend on $k$.

In view of (6), premultiplication by $U_{k}$ affects only the $i$ th and $j$ th rows of $A_{k}$. Similarly, postmultiplication by $V_{k}$ affects only the $i$ th and $j$ th columns, and elements in these rows and columns will be called affected. All other elements $a_{p q}$ are thus unaffected by the transformation; i.e. we have

$$
a_{p q}^{\prime}=a_{p q} \quad(p \neq i, j ; q \neq i, j) .
$$

Furthermore, since $U_{k}$ is unitary, premultiplication of $A_{k}$ by $U_{k}$ leaves the sum of the squares of the absolute values of the elements in each column invariant. Similarly, postmultiplication by $V_{k}$ leaves the sum of the squares of the absolute values of the elements in each row invariant. Using (12), we can thus establish the following lemma:

Lemma 1. For $p \neq i, j$,

$$
\left\{\begin{array}{l}
\left|a_{i p}^{\prime}\right|^{2}+\left|a_{j p}^{\prime}\right|^{2}=\left|a_{i p}\right|^{2}+\left|a_{j p}\right|^{2}, \\
\left|a_{p i}^{\prime}\right|^{2}+\left|a_{p j}^{\prime}\right|^{2}=\left|a_{p i}\right|^{2}+\left|a_{p j}\right|^{2} .
\end{array}\right.
$$

For brevity, we shall call the elements of the pairs $\left(a_{i p}, a_{j p}\right)$ and $\left(a_{p i}, a_{p j}\right)$, where $p \neq i, j$, coupled during the $k$ th transformation.

Using (12) and Lemma 1, it follows that

$$
S-S^{\prime}=s_{i j}-s_{i j}^{\prime} \text {. }
$$

If condition $\left(V^{\prime}\right)$ is satisfied, we have

$$
S^{\prime} \leqq S-(1-t) s_{i j}
$$

If condition ( $\left.\mathrm{I}^{\prime}\right)$ holds, then $s_{i j} \geqq 2 S n^{-1}(n-1)^{-1}$. Hence 


$$
S^{\prime} \leqq\left[1-2(1-t) n^{-1}(n-1)^{-1}\right] S .
$$

Since $0<1-q=2(1-t) n^{-1}(n-1)^{-1} \leqq 1$, we have

$$
S^{(k)} \leqq q^{k} C
$$

where $C$ is a constant and $0 \leqq q<1$. This shows that (11) is a consequence of the hypotheses of Theorem 1.

If condition $\left(\mathrm{I}^{\prime}\right)$ is not assumed to hold, we can still conclude from $\left(\mathrm{V}^{\prime}\right)$ and (14) that $0 \leqq S^{\prime} \leqq S$, and therefore that $\lim _{k \rightarrow \infty} S^{(k)}$ exists. We shall show that this limit is zero under the hypotheses of Theorem 3. Again from (14), $s_{i j} \leqq(1-t)^{-1}\left(S-S^{\prime}\right)$. From the existence of $\lim S^{(k)}$ it follows that $\lim _{k \rightarrow \infty} s_{i j}^{(k)}$ $=0$. We call the elements $a_{i j}^{(k)}$ and $a_{j}^{(k)}$, where $(i, j)=\left(i_{k}, j_{k}\right)$, the rotated elements of $A_{k}$, and state our conclusion in the following form:

Lemma 2. If condition $\left(\mathrm{V}^{\prime}\right)$ is satisfied, the rotated elements of $A_{k}$ tend to zero as $k \rightarrow \infty$.

We note that this statement does not depend on the order in which the pairs $\left(i_{k}, j_{k}\right)$ are selected. If Theorem 3 is not true, this can only be because the bulk of the quantity $S$ is pushed around in the matrix $A$ ahead of the rotated elements. Our next lemma shows that under condition $\left(I^{\prime}\right)$ an almost complete transfer of the contribution to $S$ between two coupled elements is not possible in one transformation.

Lemma 3. Suppose that $\epsilon>0$, that $p \neq i, j$ and that

$$
\left|a_{i p}\right|<\epsilon, \quad\left|a_{j p}^{\prime}\right|<\epsilon .
$$

If $U_{k}$ and $V_{k}$ satisfy $\left(\mathrm{IV}^{\prime}\right)$, then

$$
\left|a_{i p}^{\prime}\right|<C \epsilon, \quad\left|a_{j p}\right|<C \epsilon,
$$

where $C$ is a constant which depends only on the interval J. Similar statements hold if the roles of $a_{i p}$ and $a_{j p}$ are interchanged, and also for two coupled elements $\left(a_{p i}, a_{p j}\right)$.

Proof. By definition of $A_{k+1}$, for $p \neq i, j$,

$$
\begin{aligned}
& a_{i p}^{\prime}=e^{i \alpha} \cos \phi a_{i p}+e^{i \beta} \sin \phi a_{j p}, \\
& a_{j p}^{\prime}=-e^{i \gamma} \sin \phi a_{i p}+e^{i \delta} \cos \phi a_{j p} .
\end{aligned}
$$

From the second of these relations we obtain, since (IV') implies that $\cos \phi \neq 0$,

$$
a_{j p}=e^{-i \delta}\left(a_{j p}^{\prime}+e^{i \gamma} \sin \phi a_{i p}\right)(\cos \phi)^{-1} .
$$

Inserting this into the first relation and using (5), we get 


$$
a_{i p}^{\prime}=e^{i \alpha}\left(a_{i p}+e^{-i \gamma} \sin \phi a_{j p}^{\prime}\right)(\cos \phi)^{-1} .
$$

Introducing the abbreviations $c=\min _{\phi \in J} \cos \phi, s=\max _{\phi \in J}|\sin \phi|$, we find from (17) and (18) that

$$
\left|a_{j p}\right| \leqq \epsilon(1+s) c^{-1}, \quad\left|a_{i p}^{\prime}\right| \leqq \epsilon(1+s) c^{-1} .
$$

Hence (16) has been proved with

$$
C=(1+s) c^{-1} .
$$

The remaining assertions of the lemma can be proved similarly and yield the same value of $C$.

2.2. Sufficiency of $\left(\mathrm{V}^{\prime}\right)$ and $\left(I V^{\prime}\right)$ for (11). In this section we shall combine the lemmas of $\$ 2.1$ with the special orderings of the pairs given by the rules (III) to obtain Lemma 4, which will be the principal tool for the proof of (11).

We introduce some terminology. For $1 \leqq p<q \leqq n$ we shall define the submatrix $M_{p q}^{(\boldsymbol{k})}$ as follows:

$$
M_{p q}^{(k)}=\left(a_{l m}^{(k)}\right),
$$

where $p \leqq l \leqq q$ and $p \leqq m \leqq q$.

An index $k$ will be said to be associated with a pair $(p, q)$, written $k=I(p, q)$, if $\left(i_{k}, j_{k}\right)=(p, q)$. Fix one of the rules (III). A set of indices $\left(k_{1}, k_{2}, \cdots, k_{m}\right)$ will be called cocyclic, if it is contained in one of the intervals $[l N,(l+1) N-1]$, where $l=0,1,2, \cdots$, and $N=n(n-1) / 2$ is the number of rotations necessary to make up a full cycle under the fixed rule (III). For $1 \leqq p<q \leqq n$ we define

$$
S_{p q}^{(k)}=\sum_{p \leq l \leq q ; p \leq m \leq q ; l \neq m}\left|a_{l m}^{(k)}\right|^{2}
$$

We note that

$$
S_{p, p+1}^{(k)}=s_{p, p+1}^{(k)}
$$

and

$$
S_{1, n}^{(k)}=S^{(k)} \text {. }
$$

Lemma 4. Assume (IV') and ( $\left.\mathrm{V}^{\prime}\right)$ hold. Let one of the rules (III) be adopted for the selection of $\left(i_{k}, j_{k}\right)$, and let $\epsilon>0$ be given. Let $k_{0}$ be such that $s_{k j}^{(k)}<\epsilon$ for all $k>k_{0}$, and let $h, r, s$ be three cocyclic indices associated with the pairs $(p, q)$, $(p, q+1),(p+1, q+1)$, respectively $(1 \leqq p<q<n)$. Assume $h>k_{0}$. Then the inequalities

$$
S_{p q}^{(h)}<\epsilon, \quad S_{p+1, q+1}^{(s)}<\epsilon
$$

imply

$$
S_{p, q+1}^{(r)}<D \epsilon
$$


where $D=D_{q-p}$ depends only on $q-p$ and on the constant $C$ of Lemma 3 .

Proof. We shall give the proof for the rule $\left(\mathrm{III}_{c}\right)$; the proof for the rule $\left(\mathrm{III}_{r}\right)$ is analogous. All indices occurring in the proof will be cocyclic with $h$.

We first consider the history of the off-diagonal elements of $M_{p q}^{(k)}$ for $h \leqq k \leqq r$. During the rotations with indices associated with $(p, q),(p+1, q)$, $\cdots,(q-1, q)$ the elements of $M_{p q}^{(k)}$ are either unaffected or rotated or coupled among themselves. Hence, by Lemma 1 , if $k=I(1, q+1)$,

$$
S_{p q}^{(k)}<\epsilon .
$$

During the rotations of the elements in the positions $(1, q+1),(2, q+1), \cdots$, $(p-1, q+1)$ the elements of $M_{p q}^{(k)}$ are unaffected. Therefore

$$
S_{p q}^{(r)}<\epsilon,
$$

and to prove the assertion of the lemma we have only to show that the squared moduli of the elements which are in $M_{p, q+1}^{(r)}$ but not in $M_{p q}^{(r)}$ are of the order of magnitude of $\epsilon$. This is clear for the elements $a_{p, q+1}^{(r)}$ and $a_{q+1, p}^{(r)}$, because we have by assumption

$$
S_{p, q+1}^{(r)}<\epsilon .
$$

After the rotation with index $r$ the elements $a_{m, q+1}(p<m \leqq q)$ are less than $\epsilon^{1 / 2}$ in modulus, by (23). They are coupled with the elements $a_{m p}(p<m \leqq q)$ which belong to $M_{p q}^{(r)}$ and which by (25) are less than $\epsilon^{1 / 2}$ in modulus before the rotation. Thus, by Lemma 3 ,

$$
\left.\left|a_{m, q+1}^{(r)}\right|<C_{\epsilon}^{1 / 2} \quad \text { (for all } m \text { with } p<m \leqq q\right),
$$

where $C$ is given by (19). Similarly we can prove

$$
\left.\left|a_{q+1, m}^{(r)}\right|<C \epsilon^{1 / 2} \quad \text { (for all } m \text { with } p<m \leqq q\right) .
$$

Combining (25), (26), (27), and (28), we get

$$
\begin{aligned}
S_{p, q+1}^{(r)} & =S_{p q}^{(r)}+s_{p, q+1}^{(r)}+\sum_{m=p+1}^{q} s_{m, q+1}^{(r)} \\
& <\left[2+2(q-p) C^{2}\right] \epsilon .
\end{aligned}
$$

Hence Lemma 4 has been proved with

$$
D=D_{q-p}=2\left[1+(q-p) C^{2}\right] .
$$

Proof of (11). Let $\delta>0$ be given and choose $h$ such that $h=I(1,2)$, while $s_{i j}^{(k)}<\left(D_{1} D_{2} \cdots D_{n-2}\right)^{-1} \delta$ for all $k \geqq h$. Then the hypothesis of Lemma 4 is satisfied for $q-p=1$ and $\epsilon=\left(D_{1} D_{2} \cdots D_{n-2}\right)^{-1} \delta$. We use induction with respect to $q-p$. Assume that for some positive integer $l<n$ 


$$
\left.S_{p, p+l}^{(k)}<\left(D_{l} D_{l+1} \cdots D_{n-2}\right)^{-1} \delta \quad \text { (for all } p \text { such that } 1 \leqq p \leqq n-l\right),
$$

and for all $k=I(p, p+l)$ cocyclic with $h$. By Lemma 4 it then follows that

$$
S_{p, p+l+1}^{(r)}<\left(D_{l+1} \cdots D_{n-2}\right)^{-1} \delta \quad(\text { for all } p \text { such that } 1 \leqq p \leqq n-l-1),
$$

and for all $r=I(p, p+l+1)$ cocyclic with $h$. But this is (30) with $l$ replaced by $l+1$. Since $(30)$ is true for $l=1$, it follows that $S_{1, n}^{(s)}<\delta$, where $s=I(1, n)$. By (22) this is equivalent to $S^{(s)}<\delta$. Since $\delta$ was arbitrary, this establishes that the hypotheses of Theorem 3 imply (11).

2.3. Proof that (11) implies $(\mathrm{N})$. We shall establish the following lemma, in which again no reference is made to any particular property of the matrices $U_{k}$ and $V_{k}$.

Lemma 5. Any choice of the matrices $U_{k}$ and $V_{k}$ (or, if $A=A^{*}$, of $\left.U_{k}\right)$ which implies that

$$
S^{(k)} \rightarrow 0
$$$$
(k \rightarrow \infty)
$$

also implies Proposition (N).

We shall base our proof on relation (31), which is deducible from the maximum-minimum property of the eigenvalues of hermitian matrices, but which we shall prove from a theorem established by Wielandt (after its publication by Lidskir [8]). For any $n \times n$ matrix $Z=\left(z_{p q}\right)$, define $\|\cdot Z\|$ $=\left(\sum_{p, q=1}^{n}\left|z_{p q}\right|^{2}\right)^{1 / 2}$. Let $B, C, D$ be three hermitian matrices of order $n$ with their respective eigenvalues arranged in decreasing order as vectors $\beta=\left\{\beta_{p}\right\}$, $\gamma=\left\{\gamma_{p}\right\}, \delta=\left\{\delta_{p}\right\}(p=1, \cdots, n)$. Suppose $B-C=D$. Then it was proved by Wielandt $[13$, p. 110$]$ that there exists a matrix $M$ with the properties of a doubly stochastic matrix:

$$
m_{p q} \geqq 0, \quad \sum_{p=1}^{n} m_{p q}=\sum_{q=1}^{n} m_{p q}=1,
$$

such that

$$
\beta-\gamma=M \delta
$$

Hence, for any $p$,

$$
\begin{aligned}
\left|\beta_{p}-\gamma_{p}\right| & \leqq \sum_{q=1}^{n} m_{p q}\left|\delta_{q}\right| \leqq \sum_{q=1}^{n} m_{p q} \max _{q}\left|\delta_{q}\right| \\
& =\max _{q}\left|\delta_{q}\right| \leqq\left(\sum_{q=1}^{n} \delta_{q}^{2}\right)^{1 / 2}=\|D\| .
\end{aligned}
$$

The last equality is a consequence of the analogue of (1) for hermitian matrices. Hence, dropping the assumption that the eigenvalues of $B$ and $C$ are 
ordered monotonically, we see that there exists a permutation $\left\{\nu_{1}, \cdots, \nu_{n}\right\}$ of the set $\{1, \cdots, n\}$ such that

$$
\left|\beta_{p}-\gamma_{p_{p}}\right| \leqq\|B-C\| \quad(p=1, \cdots, n) .
$$
Then

To prove Lemma 5 in the hermitian case, we set $B=\left(\delta_{p q} a_{p q}^{(\mathbf{k})}\right)$ and $C=A_{k}$.

$$
\|C-B\|=\left(S^{(k)}\right)^{1 / 2} .
$$

Writing

$$
\left(S^{(k)}\right)^{1 / 2}=\epsilon_{k},
$$

and using the fact that the eigenvalues of $A_{k}$ are the same as those of $A$, we find that

$$
\left|a_{p p}^{(k)}-\lambda_{\nu_{p}(k)}\right| \leqq \epsilon_{k} \quad(p=1, \cdots, n ; k=0,1, \cdots) .
$$

The notation $\nu_{p}(k)$ indicates that the permutation $\nu_{p}$ may depend on $k$. Since $\epsilon_{k} \rightarrow 0$, (33) establishes Lemma 5 for $A=A^{*}$.

To prove the lemma in the nonhermitian case, we write

$$
A_{k}=D_{k}+E_{k},
$$

where $D_{k}=\left(\delta_{p q} a_{p q}^{(k)}\right)$. This makes

$$
\left\|E_{k}\right\|=\epsilon_{k} .
$$

We apply (31) with $B=D_{k} D_{k}^{*}$ and $C=A_{k} A_{\mathbf{k}}^{*}$. We have

$$
\|C-B\|=\left\|E_{k} D_{k}^{*}+D_{k} E_{k}^{*}+E_{k} E_{k}^{*}\right\| \leqq\left\|E_{k}\right\|\left(2\left\|D_{k}\right\|+\left\|E_{k}\right\|\right)
$$

and, in view of (34),

$$
\left\|D_{k}\right\| \leqq\left\|A_{k}\right\|+\left\|E_{k}\right\|
$$

Hence, since

$$
\begin{aligned}
\left\|A_{k}\right\| & =\|A\| \\
\|C-B\| & \leqq \epsilon_{k}\left(2\|A\|+3 \epsilon_{k}\right) .
\end{aligned} \quad(k=1,2, \cdots),
$$

Also, the eigenvalues of $C$ are the squares of the principal values of $A$. It follows that there exists a sequence of permutations

$$
\nu_{p}(k)(p=1, \cdots, n ; k=1,2, \cdots)
$$

such that

$$
\left.|| a_{p p}^{(k)}\right|^{2}-\pi_{p_{p}(k)}^{2} \mid \leqq \epsilon_{k}\left(2\|A\|+3 \epsilon_{k}\right) .
$$

Since $\epsilon_{k} \rightarrow 0$, (36) establishes Proposition (N).

At this stage we have proved Theorem 1 and also a weakened form of Theorem 3, obtained from Theorem 3 by substituting $(N)$ for $(P)$. 
2.4. Proof of Theorem 4. We now prove Theorem 4, starting with the case $A=A^{*}$. If the eigenvalues of $A$ are all identical, there is nothing left to prove. If not, set

$$
d=\min _{\lambda_{p} \neq \lambda_{q}}\left|\lambda_{p}-\lambda_{q}\right|,
$$

and define $\epsilon_{k}$ by (32). Let $k_{0}$ be such that $\epsilon_{k}<d / 2$ for all $k>k_{0}$. Then for $k>k_{0}$ every diagonal element $a_{p p}^{(\mathbf{k})}$ of $A_{k}$ is by (33) closest to exactly one number of the set $\left\{\lambda_{q}\right\}$. We call this number the eigennumber affiliated with $a_{p p}^{(\mathbf{k})}$. (The term eigennumber serves to emphasize that we no longer distinguish between identical eigenvalues.)

The proof of Theorem 4 for $A=A^{*}$ will be complete if we establish the following lemma:

Lemma 6. No diagonal element of $A_{k}$ can change its affiliation if $k$ is suffciently large.

Proof. Since the set $\left\{\nu_{p}(k)\right\}$ is a permutation of the set $\{1, \cdots, n\}$ for each $k$, if any one element changes its affiliation, at least one other element must do likewise. On the other hand, an element can change its affiliation at any particular step only if it is affected at that step. Since exactly two diagonal elements are affected at each step, it follows that a change of affiliation can take only the form of an exchange of affiliation between two elements affiliated with two different eigennumbers. Again omitting the index $k$, we let $a_{i i}$ and $a_{j j}$ be two such elements, and let them be affiliated with the eigennumbers $\mu$ and $\nu$, respectively. By (33)

$$
\left|a_{i i}-a_{j j}\right| \leqq|\mu-\nu|+2 \epsilon_{k} .
$$

By (6), the element $a_{i i}$ transforms according to

$$
a_{i i}^{\prime}=a_{i i} \cos ^{2} \phi+a_{j j} \sin ^{2} \phi+\operatorname{Re}\left[a_{i j} e^{i(\alpha-\beta)}\right] \sin 2 \phi .
$$

Hence

$$
a_{i i}-a_{i i}^{\prime}=\left(a_{i i}-a_{j j}\right) \sin ^{2} \phi-\operatorname{Re}\left[a_{i j} e^{i(\alpha-\beta)}\right] \sin 2 \phi .
$$

Using the facts that, in view of (IV), $\sin ^{2} \phi \leqq s^{2}<1$, where $s$ is independent of $k$, and that $\left|a_{i j}\right| \leqq \epsilon_{k}$, we get, using (38),

$$
\left|a_{i i}-a_{i i}^{\prime}\right| \leqq s^{2}\left(|\mu-\nu|+2 \epsilon_{k}\right)+\epsilon_{k} .
$$

If $a_{\mathfrak{s}}^{\prime}$ were affiliated with $\nu$, it would have to satisfy

$$
\left|a_{i i}^{\prime}-\nu\right| \leqq \epsilon_{k+1} .
$$

This would imply

$$
\left|a_{i i}^{\prime}-a_{i i}\right| \geqq|\mu-\nu|-\epsilon_{k}-\epsilon_{k+1} .
$$


For $k$ sufficiently large, this inequality is incompatible with (39). Hence $a_{i i}$ cannot change its affiliation. Lemma 6 and Theorem 4 for $A=A^{*}$ are thus proved.

To prove Theorem 4 in the general case, let $C_{k}=A_{k} A_{k}^{*}$, as in $\S 2.3$. We have

$$
C_{k+1}=U_{k} A_{k} V_{k} V_{k}^{*} A_{k}^{*} U_{k}^{*}=U_{k} A_{k} A_{k}^{*} U_{k}^{*}=U_{k} C_{k} U_{k}^{*} .
$$

The sequence of matrices $\left\{C_{k}\right\}$ can thus be thought of as generated by a Jacobi process for the hermitian matrix $C_{0}=A A^{*}$ and defined by the sequence of matrices $U_{k}$. Since the sequence $\left\{A_{k}\right\}$ satisfies Proposition $(\mathrm{N})$, the sequence $\left\{C_{k}\right\}$ satisfies the hermitian property $(\mathrm{N})$. The angles $\phi_{k}$ of $U_{k}$ satisfy $\left(\mathrm{IV}^{\prime}\right)$ and hence (IV). The sequence $\left\{C_{k}\right\}$ is thus generated by a hermitian Jacobi process satisfying (N) and (IV). By the statement of Theorem 4 for hermitian matrices, which has been proved above, $\left\{C_{k}\right\}$ converges to a diagonal matrix. The diagonal elements of this matrix are the squares of the principal values of $A$ in some order. Thus

$$
c_{p p}^{(k)}=\sum_{q=1}^{n}\left|a_{p q}^{(k)}\right|^{2} \rightarrow \pi_{p p}^{2} \quad(k \rightarrow \infty ; p=1, \cdots, n),
$$

where $\left\{\nu_{p}\right\}$ is some fixed permutation of $\{1, \cdots, n\}$. But

$$
\sum_{q=1 ; q \ngtr p}^{n}\left|a_{p q}^{(k)}\right|^{2} \leqq \epsilon_{k}^{2} .
$$

Hence

$$
\left|a_{p p}^{(k)}\right| \rightarrow \pi_{p p} \quad(k \rightarrow \infty ; p=1, \cdots, n) .
$$

This completes the proof of the first sentence of Theorem 4.

To prove that $\left(\mathrm{IV}^{\prime}\right)$ is necessary for the validity of Theorem 4 , let

$$
A_{0}=\left(\begin{array}{rr}
1 & 0 \\
0 & -1
\end{array}\right)
$$

and choose

$$
\phi_{k}=\pi / 2 \quad(k=0,1, \cdots) .
$$

Then

$$
A_{k}=\left(\begin{array}{rr}
-1 & 0 \\
0 & 1
\end{array}\right) \quad(k=1,3,5, \cdots),
$$

while $A_{k}=A_{0}(k=2,4, \cdots)$.

The following example $\left(^{4}\right)$ shows that it is impossible to replace the inter-

() See footnote 3. 
val $J$ in condition $\left(\mathrm{IV}^{\prime}\right)$ even by the open interval $(-\pi / 2, \pi / 2)$. Let $\left\{\boldsymbol{\epsilon}_{k}\right\}$ be a sequence of positive angles which converge to zero monotonically, with $\epsilon_{0}<\pi / 2$. Put

$$
A_{0}=\left(\begin{array}{rr}
\cos \epsilon_{0} & -\sin \epsilon_{0} \\
-\sin \epsilon_{0} & -\cos \epsilon_{0}
\end{array}\right)
$$

and choose

$$
U_{k}=\left(\begin{array}{rr}
\cos \phi_{k} & \sin \phi_{k} \\
-\sin \phi_{k} & \cos \phi_{k}
\end{array}\right)
$$

where $\phi_{k}=(-1)^{k}\left(\pi-\epsilon_{k}-\epsilon_{k+1}\right) / 2$. It is easily verified that

$$
A_{k}=\left(\begin{array}{cc}
(-1)^{k} \cos \epsilon_{k} & -\sin \epsilon_{k} \\
-\sin \epsilon_{k} & (-1)^{k+1} \cos \epsilon_{k}
\end{array}\right) .
$$

Obviously, the sequence $\left\{A_{k}\right\}$ satisfies (11), and the angles $\phi_{k}$ all lie in $(-\pi / 2, \pi / 2)$. Yet the sequence $\left\{A_{k}\right\}$ does not converge to a diagonal matrix.

This completes the proof of Theorem 4. As we remarked above, out of Theorem 4 now follows the truth of Theorem 2 and of the sufficiency part of Theorem 3.

2.5. Necessity of condition $\left(I V^{\prime}\right)$ for Theorem 3. To prove that condition $\left(I^{\prime}\right)$ is necessary for the convergence of the cyclic Jacobi method even in the weaker sense of Proposition (N), we shall exhibit a real symmetric matrix for which (11) fails to hold, if the angles $\phi_{k}$ are only subjected to (II). Obviously the order of such a matrix must be at least 3 . We shall achieve divergence by constructing a matrix for which $a_{i j}^{(k)}=0$ for all $k$ and by then selecting $\phi_{k}=\pi / 2$. This interchanges the two affected elements in the main diagonal and does not destroy the zero in the $(i, j)$-position. If the ordering $\left(\mathrm{III}_{r}\right)$ is adopted, a matrix with the desired features is given by

$$
A=A_{0}=\left(\begin{array}{lll}
2 & 0 & 1 \\
0 & 3 & 0 \\
1 & 0 & 4
\end{array}\right) \text {. }
$$

It is easily verified that

$$
\begin{array}{rlrl}
A_{1}= & \left.\begin{array}{rrr}
3 & 0 & 0 \\
0 & 2 & -1 \\
0 & -1 & 4
\end{array}\right), & A_{2}=\left(\begin{array}{rrr}
4 & -1 & 0 \\
-1 & 2 & 0 \\
0 & 0 & 3
\end{array}\right), & A_{3}=\left(\begin{array}{lll}
4 & 0 & 1 \\
0 & 3 & 0 \\
1 & 0 & 2
\end{array}\right), \\
A_{4}=\left(\begin{array}{rrr}
3 & 0 & 0 \\
0 & 4 & -1 \\
0 & -1 & 2
\end{array}\right), & A_{5}=\left(\begin{array}{rrr}
2 & 0 & 1 \\
-1 & 4 & 0 \\
0 & 0 & 3
\end{array}\right), & A_{6}=\left(\begin{array}{lll}
0 & 3 & 0 \\
1 & 0 & 4
\end{array}\right) .
\end{array}
$$


Thus $A_{6}=A_{0}$ and hence $A_{k+6}=A_{k}$ for all $k$, so that the sequence $\left\{A_{k}\right\}$ can never converge to a diagonal matrix.

REMARK. From the point of view of numerical computation it might seem unfair to perform a nonzero rotation when the element to be annihilated is already zero. Actually, we have also constructed examples of matrices where the cyclic Jacobi method fails even if $\phi_{k}$ is selected such that (II) holds and $0 \leqq \phi_{k}<\pi / 2$. This will be the case for any matrix of the form

$$
A_{0}=\left(\begin{array}{ccc}
a & \epsilon & 1 \\
\epsilon & a+c & 0 \\
1 & 0 & a+2 c
\end{array}\right),
$$

where $a$ is arbitrary, $c>0$ is sufficiently large and $\epsilon(>0)$ is sufficiently small. (We have proved that (11) fails for $c \geqq 4$ and $\epsilon \leqq 1$.) This example also shows that it is not possible to replace the interval $J$ in condition (IV) by the open interval $(-\pi / 2, \pi / 2)$.

\section{Realization OF THE CONDITIONS OF TheOREMS 1 TO 4}

In this section we shall justify the statements made in $\$ 1.2$ concerning the existence of unitary matrices $U_{k}$ and $V_{k}$ satisfying the conditions of Theorems 1, 2, 3, and 4, both for an arbitrary complex matrix $A$ and for $A=A^{*}$ (in the latter case we assume $V_{k}=U_{k}^{*}$ ). Throughout this section we put

$$
a_{p q}=a_{p q}^{(k)}, \quad a_{p q}^{\prime}=a_{p q}^{(k+1)}
$$

and omit the subscript $k$ on the parameters $\alpha, \beta, \gamma, \delta, \phi$, and $\xi, \eta, \zeta, \omega, \psi$. We shall also write

$$
a_{p q}=r_{p q} e^{i \theta_{p q}},
$$

where $r_{p q}$ and $\theta_{p q}$ are real and $r_{p q} \geqq 0$.

3.1. Eigenvalues of hermitian matrices. We assume that $A=A^{*}$ and $V_{k}=U_{k}^{*}$ for all $k$. The latter condition implies

$$
\xi \equiv-\alpha, \quad \eta \equiv-\gamma, \quad \zeta \equiv-\beta, \quad \omega \equiv-\delta
$$

and

$$
\psi \equiv-\phi,
$$

where all congruences are taken modulo $2 \pi$.

Theorem 5. Let $A=A^{*}$ and $A_{k+1}=U_{k} A U_{k}^{*}$. In order that condition (II) be satisfied it is necessary and sufficient that the parameters of $U_{k}$ satisfy the following relations:(5)

(5) Condition (43) should be disregarded when $r_{i j}=0$. Condition (44) and all subsequent tangent equations should be disregarded when they take the form $\tan \mu=0 / 0$. 


$$
\begin{aligned}
\beta-\alpha & \equiv \theta_{i j}(\bmod \pi), \\
\tan 2 \phi & = \pm 2 r_{i j}\left(a_{i i}-a_{j j}\right)^{-1} .
\end{aligned}
$$

The upper or lower sign is to be chosen according to whether $\beta-\alpha$ differs from $\theta_{i j}$ by an even or an odd multiple of $\pi$, respectively.

Proof. Direct computation yields, if $U_{k}$ is any matrix of the form (6), $a_{i j}^{\prime}=\bar{a}_{j i}^{\prime}=\cos \phi \sin \phi\left(e^{i(\beta-\delta)} a_{j j}-e^{i(\alpha-\gamma)} a_{i i}\right)+\cos ^{2} \phi e^{i(\alpha-\delta)} a_{i j}-\sin ^{2} \phi e^{i(\beta-\gamma)} a_{j i}$. Hence, using (5),

$$
\begin{aligned}
e^{i(\delta-\beta)} a_{i j}^{\prime} & =\sin 2 \phi\left(a_{j j}-a_{i i}\right) / 2+\cos ^{2} \phi e^{i(\alpha-\beta)} a_{i j}-\sin ^{2} \phi e^{-i(\alpha-\beta)} \bar{a}_{i j} \\
& =\sin 2 \phi\left(a_{j j}-a_{i i}\right) / 2+\operatorname{Re}\left[e^{i(\alpha-\beta)} a_{i j}\right] \cos 2 \phi+i \operatorname{Im}\left[e^{i(\alpha-\beta)} a_{i j}\right] .
\end{aligned}
$$

If (II) is satisfied, the expression on the right side of the last equation is zero. Putting its real and imaginary parts equal to zero yields the necessity of (43) and (44). Their sufficiency is obvious from (45).

If $A$ is a real symmetric matrix, an obvious solution of (43) is $\alpha=\beta=\gamma=\delta$ $=0$. It follows that in this case $U_{k}$ may be taken to be a real orthogonal matrix. Equation (44) then takes the form given by Jacobi,

$$
\tan 2 \phi=2 a_{i j}\left(a_{i i}-a_{j j}\right)^{-1} \text {. }
$$

Returning to hermitian $A$, we note that, since the function $\tan 2 \phi$ takes on all real values in each closed interval of length $\pi / 2, \phi$ can be selected to satisfy (IV), provided the length of $J$ is at least $\pi / 2$. It follows that for a hermitian matrix $A$ conditions (IV) and (V) can always be realized with $t=0$.

By the above it is trivial that for $A=A^{*}$ conditions (IV) and (V) can be realized for every $t>0$. Conversely, we shall now show that even if (44) is not completely satisfied (V) may still hold with some $t<1$.

We assume that (43) holds and define $\rho$ by

$$
\tan 2 \rho= \pm 2 r_{i j}\left(a_{i i}-a_{j j}\right)^{-1}, \quad-\pi / 4 \leqq \rho \leqq \pi / 4,
$$

with the sign convention of Theorem 5 . We then have

Theorem 6. Let $A=A^{*}$ and $V_{k}=U_{k}^{*}$. If (43) holds and if

$$
\phi=(1-p) \rho,
$$

where $-1<p<1$, then cand $(\mathrm{V})$ is satisfied with

$$
t=\sin ^{2}(p \pi / 2) \text {. }
$$

Proof. We shall make use of the following two facts, both of which are easily verified:

(i) If $-1<p<1$ and 


$$
g(x)=\left\{\begin{array}{l}
p \\
\sin p x / \sin x
\end{array}\right.
$$

$$
\begin{array}{r}
(x=0), \\
(0<|x| \leqq \pi / 2),
\end{array}
$$

then

$$
\max _{|x| \leqq \pi / 2}|g(x)|=|\sin (p \pi / 2)|
$$

(ii) If $a, b$ are real, and

$$
\tan \phi=b / a,
$$

then

$$
\left(a^{2}+b^{2}\right) \sin ^{2} \phi=b^{2} .
$$

Turning to the proof of Theorem 6, we find from (43), (45), and (47) that

$$
\left|a_{i j}^{\prime}\right|^{2}=\left[\left(a_{j j}-a_{i i}\right)^{2} / 4+r_{i j}^{2}\right] \sin ^{2}(2 \phi-2 \rho) \text {. }
$$

Then by (49) and (50)

$$
\begin{aligned}
\left|a_{i j}^{\prime}\right|^{2} & =\left[\left(a_{j j}-a_{i i}\right)^{2} / 4+r_{i j}^{2}\right] \sin ^{2} 2 p \rho \\
& \leqq \sin ^{2}(p \pi / 2)\left[\left(a_{j j}-a_{i i}\right)^{2} / 4+r_{i j}^{2}\right] \sin ^{2} 2 \rho \\
& =\sin ^{2}(p \pi / 2) r_{i j}^{2} .
\end{aligned}
$$

This completes the proof of Theorem 6.

3.2. Principal values of arbitrary complex matrices. We shall now formulate and prove two theorems which are analogous to those of $\$ 3.1$ when $A$ is an arbitrary complex matrix and $U_{k}$ and $V_{k}$ are not related. It is convenient to perform some preliminary computations before stating the analogue of Theorem 6.

If $U_{k}$ and $V_{k}$ are arbitrary matrices of the forms (6) and (7), we find for the rotated elements of $A_{k+1}$ the following expressions:

$$
\begin{aligned}
a_{i i}^{\prime}= & e^{i(\alpha+\xi)} \cos \phi \cos \psi a_{i i}-e^{i(\alpha+\zeta)} \cos \phi \sin \psi a_{i j} \\
& +e^{i(\beta+\xi)} \sin \phi \cos \psi a_{j i}-e^{i(\beta+\zeta)} \sin \phi \sin \psi a_{j j} ; \\
a_{i j}^{\prime}= & e^{i(\alpha+\eta)} \cos \phi \sin \psi a_{i i}+e^{i(\alpha+\omega)} \cos \phi \cos \psi a_{i j} \\
& +e^{i(\beta+\eta)} \sin \phi \sin \psi a_{j i}+e^{i(\beta+\omega)} \sin \phi \cos \psi a_{j j} ; \\
a_{j i}^{\prime}= & -e^{i(\gamma+\xi)} \sin \phi \cos \psi a_{i i}+e^{i(\gamma+\zeta)} \sin \phi \sin \psi a_{i j} \\
& +e^{i(\delta+\xi)} \cos \phi \cos \psi a_{j i}-e^{i(\delta+\zeta)} \cos \phi \sin \psi a_{j j} ; \\
a_{j j}^{\prime}= & -e^{i(\gamma+\eta)} \sin \phi \sin \psi a_{i i}-e^{i(\gamma+\omega)} \sin \phi \cos \psi a_{i j} \\
& +e^{i(\delta+\eta)} \cos \phi \sin \psi a_{j i}+e^{i(\delta+\omega)} \cos \phi \cos \psi a_{j j} .
\end{aligned}
$$

Multiplying the third equation by $e^{i \lambda}$, where $\lambda$ is a real number to be 
determined later, and adding it to the second equation, we obtain

$$
\begin{aligned}
a_{i j}^{\prime}+e^{i \lambda} a_{j i}^{\prime}= & a_{i i}\left(e^{i(\alpha+\eta)} \cos \phi \sin \psi-e^{i(\gamma+\xi+\lambda)} \sin \phi \cos \psi\right) \\
& +a_{i j}\left(e^{i(\alpha+\omega)} \cos \phi \cos \psi+e^{i(\gamma+\zeta+\lambda)} \sin \phi \sin \psi\right) \\
& +a_{j i}\left(e^{i(\beta+\eta)} \sin \phi \sin \psi+e^{i(\delta+\xi+\lambda)} \cos \phi \cos \psi\right) \\
& +a_{j j}\left(e^{i(\beta+\omega)} \sin \phi \cos \psi-e^{i(\delta+\zeta+\lambda)} \cos \phi \sin \psi\right) .
\end{aligned}
$$

We now select $\lambda=\alpha-\gamma-\xi+\eta$. Since

$$
\alpha-\beta-\gamma+\delta \equiv \xi-\eta-\zeta+\omega \equiv 0(\bmod 2 \pi),
$$

our choice of $\lambda$ implies that

$$
\lambda \equiv \beta-\delta-\zeta+\omega(\bmod 2 \pi) .
$$

It follows that (51) can be written in the form

$$
a_{i j}^{\prime}+\epsilon a_{j i}^{\prime}=C \sin (\phi-\psi)+D \cos (\phi-\psi),
$$

where $|\epsilon|=1$ and

$$
\begin{aligned}
& C=a_{j j} e^{i(\beta+\omega)}-a_{i i} e^{i(\alpha+\eta)}, \\
& D=a_{i j} e^{i(\alpha+\omega)}+a_{j i} e^{i(\beta+\eta)} .
\end{aligned}
$$

Similarly, we obtain

$$
a_{i j}^{\prime}-\epsilon a_{j i}^{\prime}=E \sin (\phi+\psi)+F \cos (\phi+\psi),
$$

where

$$
\begin{aligned}
& E=a_{j j} e^{i(\beta+\omega)}+a_{i i} e^{i(\alpha+\eta)}, \\
& F=a_{i j} e^{i(\alpha+\omega)}-a_{j i} e^{i(\beta+\eta)} .
\end{aligned}
$$

Using the abbreviations

$$
\begin{array}{lll}
\Theta_{\Delta p}=\theta_{i p}-\theta_{j p}, & \Theta_{p \Delta}=\theta_{p i}-\theta_{p j}, & \\
R_{x p}=r_{i p} r_{j p}, & R_{p \pi}=r_{p i} r_{p j} & (p=i, j),
\end{array}
$$

we now can state the following result:

THEOREM $5^{\prime}$. In order that the unitary matrices $U_{k}$ and $V_{k}$ satisfy condition $\left(\mathrm{II}^{\prime}\right)$, it is necessary and sufficient that their parameters obey the following relations:

$$
\begin{aligned}
& \tan (\alpha-\beta)=-\frac{R_{\pi i} \sin \Theta_{\Delta i}+R_{\pi j} \sin \Theta_{\Delta j}}{R_{\pi i} \cos \Theta_{\Delta i}+R_{\pi j} \cos \Theta_{\Delta j}}, \\
& \tan (\eta-\omega)=-\frac{R_{i \pi} \sin \Theta_{i \Delta}+R_{j \pi} \sin \Theta_{j \Delta}}{R_{i \pi} \cos \Theta_{i \Delta}+R_{\pi} \cos \Theta_{j \Delta}},
\end{aligned}
$$




$$
\tan (\phi-\psi)=-D / C, \quad \tan (\phi+\psi)=-F / E,
$$

where $C, D, E, F$ are defined in (54) and (56).

Proof. We assume that condition $\left(\mathrm{II}^{\prime}\right)$ is satisfied. Then the left sides of (53) and (55) are zero. The condition that the numbers $\sin (\phi \pm \psi)$ and $\cos (\phi \pm \psi)$ be real is then equivalent to the condition that $C \bar{D}$ and $E \bar{F}$ be real. Expressing the fact that $\operatorname{Im}(C \bar{D} \pm E \bar{F})=0$, we readily obtain the equations (57). Conversely, if conditions (57) are met, $C \bar{D}$ and $E \bar{F}$ are real numbers. If ( $\left.\mathrm{II}^{\prime}\right)$ holds, then the equations (53) and (55) imply (58). Conversely, if (58) holds, then $a_{i j}^{\prime}$ and $a_{f t}^{\prime}$ are zero. This completes the proof of Theorem $5^{\prime}$.

If the matrix $A$ is real, an obvious solution of (57) is $\alpha=\beta=\eta=\omega=0$. We then may also take $\gamma=\delta=\xi=\zeta=0$. It follows that in this case $U_{k}$ and $V_{k}$ may be assumed to be real orthogonal matrices. The equations (58) then take the form

$$
\begin{aligned}
& \tan (\phi-\psi)=\left(a_{j i}+a_{i j}\right) /\left(a_{i i}-a_{j j}\right), \\
& \tan (\phi+\psi)=\left(a_{j i}-a_{i j}\right) /\left(a_{i i}+a_{j j}\right) .
\end{aligned}
$$

When $A$ is real and symmetric, we may take $V_{k}=U_{k}^{*}$, so that $\phi=-\psi$. Note that the first condition (59) then reduces to (46).

Returning to the general case, we shall now show that the conditions $\left(\mathrm{II}^{\prime}\right)$ and $\left(\mathrm{IV}^{\prime}\right)$ cannot always be realized simultaneously. By (58) the values of $\phi \pm \psi$ are determined modulo $\pi$. Let

$$
\phi-\psi=\chi+n \pi, \quad \phi+\psi=\kappa+m \pi,
$$

where $m$ and $n$ are integers to be chosen suitably. It follows that

$$
\begin{aligned}
& \phi=(\kappa+\chi) / 2+(m+n) \pi / 2, \\
& \psi=(\kappa-\chi) / 2+(m-n) \pi / 2 .
\end{aligned}
$$

We can select $k=m+n$ such that $\phi \in J$. There are values of $\kappa+\chi$ for which there is only one possible choice of $k$. It follows that

$$
\psi=(\kappa-\chi) / 2+k \pi / 2-n \pi .
$$

Without changing $\kappa+\chi$, we can adjust $\kappa-\chi$ so that there is no $n$ for which $\psi \in J$.

The next theorem implies the fact that for any $t>0$ the conditions (IV') and $\left(\mathrm{V}^{\prime}\right)$ can be realized simultaneously.

We define $\sigma$ and $\tau$ by

$$
\begin{array}{cc}
\tan \tau=-D / C, & \tan \sigma=-F / E, \\
-\pi / 2 \leqq \tau \leqq \pi / 2, & -\pi / 2 \leqq \sigma \leqq \pi / 2 .
\end{array}
$$

Theorem $6^{\prime}$. If (47) holds and if 


$$
\phi=(1-p)(\sigma+\tau) / 2, \quad \psi=(1-p)(\sigma-\tau) / 2,
$$

where $-1<p<1$, then condition $\left(\mathrm{V}^{\prime}\right)$ holds with

$$
t=\sin ^{2}(p \pi / 2) \text {. }
$$
real,

Proof. From (53) and (55) we have, using the fact that $C \bar{D}$ and $E \bar{F}$ are

$$
\begin{aligned}
s_{i j}^{\prime} & =\left|a_{i j}^{\prime}\right|^{2}+\left|a_{j i}^{\prime}\right|^{2} \\
& =\left\{\left|a_{i j}^{\prime}+\epsilon a_{j i}^{\prime}\right|^{2}+\left|a_{i j}^{\prime}-\epsilon a_{j i}^{\prime}\right|^{2}\right\} / 2 \\
& =\left\{\left(|C|^{2}+|D|^{2}\right) \sin ^{2} p \tau+\left(|E|^{2}+|F|^{2}\right) \sin ^{2} p \sigma\right\} / 2 .
\end{aligned}
$$

As in the proof of Theorem 6 we find that

It follows that

$$
\begin{aligned}
& \left(|C|^{2}+|D|^{2}\right) \sin ^{2} p \tau \leqq \sin ^{2}(p \pi / 2)|D|^{2}, \\
& \left(|E|^{2}+|F|^{2}\right) \sin ^{2} p \sigma \leqq \sin ^{2}(p \pi / 2)|F|^{2} .
\end{aligned}
$$

$$
s_{i j}^{\prime} \leqq \sin ^{2}(p \pi / 2)\left(|D|^{2}+|F|^{2}\right) / 2=\sin ^{2}(p \pi / 2) s_{i j},
$$

proving the theorem.

For $0<p<1, \phi$ and $\psi$ as given by (61) are the arithmetic means of the number pairs $(1-p) \sigma,(1-p) \tau$, and $(1-p) \sigma,-(1-p) \tau$, respectively, both of which are contained in the interval $J=[-(1-p) \pi / 2,(1-p) \pi / 2]$. This interval meets the requirements of condition $\left(\operatorname{IV}^{\prime}\right)$. Since for every $t \in(0,1)$ there exists a $p \in(0,1)$ such that $(62)$ holds, it follows that the conditions $\left(I V^{\prime}\right)$ and $\left(V^{\prime}\right)$ can be realized simultaneously for every $t \in(0,1)$.

Professor M. R. Hestenes has remarked to us that for an arbitrary complex matrix $A$ it is possible to choose the parameters of the $U_{k}$ and the $V_{k}$ so that the limit matrix $\Pi$ of $\$ 1.2$ has only non-negative elements in its principal diagonal-i.e., the principal values of $A$ in some order. With such choices the matrices $\left|A_{k}\right|$ of Proposition (P) can be replaced by $A_{k}$, and we can assert that

$$
P A_{k} P^{-1} \rightarrow \Pi \quad(k \rightarrow \infty) .
$$

To prove Professor Hestenes' remark, refer to the formulas for $a_{\mathfrak{t}}^{\prime}$ and $a_{j \jmath}^{\prime}$ at the start of $\$ 3.2$. Observe that $a_{\mathfrak{i}}^{\prime}$ and $a_{j \jmath}^{\prime}$ can be made non-negative by changing $\alpha, \beta, \gamma, \delta$ to $\alpha+\mu, \beta+\mu, \gamma+\nu, \delta+\nu$, respectively, for some real numbers $\mu, \nu$. Since these changes do not alter conditions (5), (57), (58), (60), (61), they have no effect on $\phi, \psi, \lambda$, nor on $\left(\mathrm{II}^{\prime}\right),\left(\mathrm{IV}^{\prime}\right),\left(\mathrm{V}^{\prime}\right)$. Thus Theorem 3 continues to hold. But, since after the first cycle all $a_{\sharp}^{(k)}$ remain non-negative, the limit matrix $I$ is non-negative.

\section{Open questions}

One obvious problem which arises in connection with the results of the present paper is to find the rate of convergence of the cyclic Jacobi method. 
For the generalized Jacobi method described in Theorem 1 the rate of convergence is easily bounded by use of (14). Other questions are suggested by the strong resemblance which several aspects of the Jacobi method bear to relaxation methods for solving systems of linear equations. Ostrowski's work [11] on relaxation methods suggests, among others, the following question. Does the convergence of the Jacobi method under the conditions (II) (or (V)) and (IV) persist if (III) is replaced by any cyclic order of the couples $(i, j)$ in which all couples actually occur? How about an order which is subject only to the condition that each couple $(i, j)$ occurs infinitely of ten in the sequence $\left(i_{k}, j_{k}\right)$ ? Young's work [14] on the successive overrelaxation method raises the question whether the convergence of the cyclic Jacobi method can be improved, at least for certain matrices $A$, by systematic use of over- or underrelaxation $(p<0$ or $p>0$ in Theorem 6$)$.

\section{BIBLIOGRAPHY}

1. Robert L. Causey, Computing eigenvalues of non-hermitian matrices by methods of Jacobi type, J. Soc. Indust. Appl. Math. vol. 6 (1958) pp. 172-181.

2. Wallace Givens, Numerical computation of the characteristic values of a real symmetric matrix, Oak Ridge National Laboratory, Report ONRL 1574, February, 1954, 107 pp. (Purchasable from the Office of Technical Services, U. S. Dept. of Commerce.)

3. H. H. Goldstine, F. J. Murray, and J. von Neumann, The Jacobi method for real symmetric matrices, J. Assoc. Comput. Mach. vol. 6 (1959) pp. 59-96.

4. J. Greenstadt, $A$ method for finding roots of arbitrary matrices, Math. Tables Aids Comput. vol. 9 (1955) pp. $47-52$.

5. Robert T. Gregory, Computing eigenvalues and eigenvectors of a symmetric matrix on the ILLIAC, Math. Tables Aids Comput. vol. 7 (1953) pp. 215-220.

6. C. G. J. Jacobi, Über ein leichtes Verfahren, die in der Theorie der Säkularstörungen vorkommenden Gleichungen numerisch aufzulösen, J. Reine Angew. Math. vol. 30 (1846) pp. 51-95.

7. E. G. Kogbetliantz, Solution of linear equations by diagonalization of coefficients matrix, Quart. Appl. Math. vol. 13 (1955) pp. 123-132.

8. V. B. Lidskiy, The proper values of the sum and product of symmetric matrices (Russian), Dokl. Akad. Nauk SSSR. vol. 75 (1950) pp. 769-772.

9. Mark Lotkin, Characteristic values of arbitrary matrices, Quart. Appl. Math. vol. 14 (1956) pp. 267-275. $110 \mathrm{pp}$.

11. A. M. Ostrowski, On the linear iteration procedures for symmetric matrices, Univ. Roma. Ist. Naz. Alta Mat. Rend. Mat. e Appl. (5) vol. 13 (1954) pp. 140-163.

12. David A. Pope and C. Tompkins, Maximizing functions of rotations-experiments concerning speed of diagonalization of symmetric matrices using Jacobi's method, J. Assoc. Comput. Mach. vol. 4 (1957) pp. 459-466.

13. Helmut Wielandt, An extremum property of sums of eigenvalues, Proc. Amer. Math. Soc. vol. 6 (1955) pp. 106-110.

14. David Young, Iterative methods for solving partial difference equations of elliptic type, Trans. Amer. Math. Soc. vol. 76 (1954) pp. 92-111.

STANFORD UNIVERSITY, Stanford, California

University of CALIFornia, Los Angeles, California 